\title{
Design of an OFDM baseband receiver with space diversity
}

\author{
M.-L. Liou, P.-H. Lin, C.-J. Jan, S.-C. Lin and T.-D. Chiueh
}

\begin{abstract}
An orthogonal frequency division multiplexing (OFDM) baseband receiver chip is proposed. Carrier frequency synchronisation, symbol boundary estimation, and channel estimation and frequency-domain equalisation, all based on well known and proven algorithms, have been integrated in the receiver. The complexity and power consumption of the chip are minimised by full-custom memory blocks, word-length optimisation and innovative architecture. This chip has a core area of $4.1 \mathrm{~mm} \times 4.6 \mathrm{~mm}$. When running at $20 \mathrm{MHz}$, the chip consumes $66 \mathrm{~mW}$ from a $2.3 \mathrm{~V}$ supply. By using two such ICs and some diversity combining hardware, competitive measured packet error rates have been obtained, indicating that the chip, and the diversity receiver based on this chip, can operate reliably in a multipath fading environment.
\end{abstract}

\section{Introduction}

Wireless local area network (WLAN) has received much attention recently. Interest in wireless communication has also produced several extensive studies in communication and very large scale integration (VLSI) technologies, such as channel bandwidth utilisation, low-power dissipation, high-speed processors, and so on. Wireless communication quality is determined by several factors, including multipath fading, accuracy of local oscillator, noise and interference. Due to the impairment to communication quality and reliability, we have to pay more attention to the system design and implementation process [1].

Orthogonal frequency division multiplexing (OFDM) attracts a lot of interest because it can efficiently reduce frequency selective fading [2, 3]. This is achieved by increasing the symbol duration for the lower-rate parallel subcarriers transmission, so that each subcarrier suffers only flat fading. The IEEE 802.11a/g WLAN standard [4] adopts OFDM modulation and is designed to operate at the unlicensed bands at $2.4 \mathrm{GHz}$ and above $5 \mathrm{GHz}$. The data rates supported vary from $6 \mathrm{Mbps}$ to $54 \mathrm{Mbps}$. The highest data rate is similar to that of wired networks and is suitable for short-range data transmission.

Besides accurate functional operation, low power consumption is a very critical issue for a portable device. It is for extending battery life, reducing the cooling related cost and increasing the system reliability [5]. Although some 802.11a receiver chips for high speed WLAN applications have been reported [6, 7], low-power design with customised flow and performance comparison are not available. This paper aims at designing a low-power OFDM baseband receiver IC based on our developed design methodology [8]

(C) The Institution of Engineering and Technology 2006

IEE Proceedings online no. 20050148

doi:10.1049/ip-com:20050148

Paper first received 10th April and in revised form 13th July 2005

The authors are with the Graduate Institute of Electronics Engineering and the Department of Electrical Engineering, National Taiwan University, Taipei, Taiwan 10617, Republic of China

E-mail: chiueh@cc.ee.ntu.edu.tw and building a high-performance space-diversity WLAN receiver based on the designed IC.

The proposed chip has the following features:

- $0.35 \mu \mathrm{m}$ CMOS baseband OFDM receiver

- operates at $20 \mathrm{MHz}$ at a supply voltage of 2.3-3.3 V

- adjustable symbol boundary estimation range for different environments

- low-power and low-complexity latch-based delay buffer

- high-speed, low-latency complex reciprocal module.

In addition, by using two antennas and two receivers and properly combining the received baseband signals from these two antennas, the combined signal can be more robust even under link conditions with severe multipath fading. Increasing diversity order implies linear growth in receiver complexity (including the number of antennas, analogue front-end and baseband chip die size). From the system design perspective, it is natural to use more receiving antennas to boost the received signal quality.

In this paper, we show the implementation of an OFDM baseband receiver that achieves space diversity by using two simple baseband receiver chips (one for each antenna) and a signal processing module built in field-programmable gate array (FPGA). The receiver architecture and practical implementation aspect are the main focus and will be described in more detail.

\section{Baseband receiver for OFDM wireless LAN}

In this section we present the OFDM WLAN standard and the major tasks and scheduling of the OFDM WLAN baseband receiver.

\subsection{OFDM wireless LAN}

OFDM is a modulation scheme that splits data into parallel streams and transmits them over a number of orthogonal subcarriers. A discrete-time formulation of the transmitter is equivalent to an inverse discrete Fourier transform on the data [9] as shown in Fig. 1. After the inverse DFT processing, a cyclic prefix is inserted in each symbol to mitigate intersymbol interference (ISI) and make the received signal easy to equalise. One of the salient features 


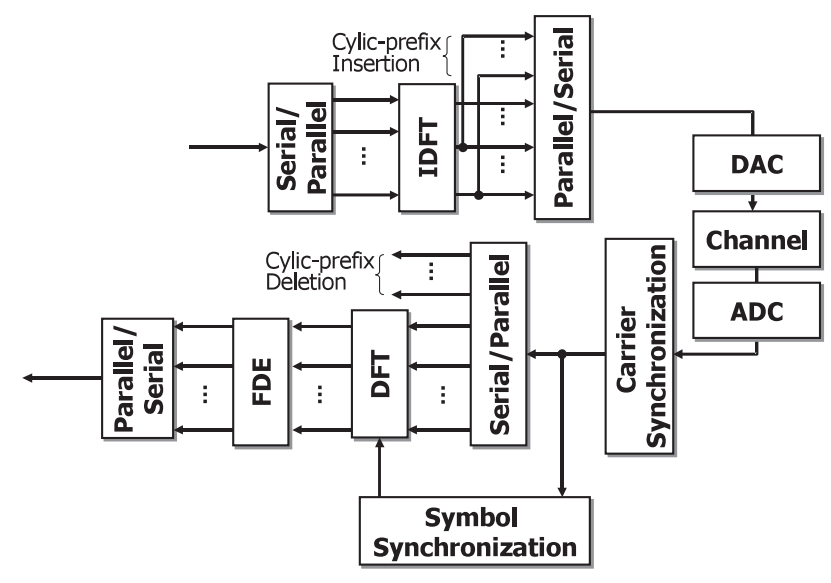

Fig. 1 Block diagram of OFDM communication systems

of OFDM technology is robustness against frequencyselective fading since corrupted data carried on faded subcarriers can be reconstructed by error correcting code. Nonetheless, OFDM systems are sensitive to carrier/timing offset and phase noise [10]. Therefore, synchronisation is a crucial issue in OFDM receiver design.

An IEEE 802.11a/g WLAN standard compliant packet that adopts OFDM contains three fields, preamble field, SIGNAL field and data field. The preamble is used for gain control, synchronisation and channel estimation. The SIGNAL field contains coding rate and modulation type. The data field carries user data. The autocorrelation property of the short preamble and the long preamble is similar to that of pseudonoise (PN) codes, making it possible to do accurate timing/channel profile estimation as in spread-spectrum systems [11]. The data symbol of each subcarrier uses either quaternary phase shift keying (QPSK), 16-point quadrature amplitude modulation (16-QAM), or 64-QAM.

\subsection{Synchronisation and equalisation}

To align frequency and avoid intercarrier interference (ICI), it is necessary to compensate the carrier frequency offset (CFO) before the receiver DFT operation. Usually delayed autocorrelation is used for detecting the carrier frequency offset and also the periodic structure of the short preamble. Furthermore, the magnitude of the delayed autocorrelation signal can be used to detect the boundary between short and long preamble segments.

To minimise ISI, the receiver tries to locate the first significant path of the channel impulse response (CIR) and determines the DFT window accordingly. One such technique transforms the frequency-domain channel response to time domain with an additional IDFT block [12]. To avoid long latency, the proposed receiver estimates the symbol boundary in the time domain using a bank of correlators matched to the long preamble. Since the long preamble is PN-code-like, the correlator outputs approximate the CIR, from which the DFT window can be derived.

The channel effect is compensated by multiplying each subcarrier data with the reciprocal of the corresponding channel gain on each subcarrier. For simplicity the gain of the $n$th subcarrier is derived using the simple least-square criteria

$$
\widehat{H}_{n}=R_{n}^{-1} L_{n}
$$

where $\widehat{H}_{n}, R_{n}$ and $L_{n}$ are the channel gain of the $n$th subcarrier, the transmitted and received $n$th subcarrier of the long preamble symbol, respectively. Finally, the equalised frequency-domain symbols are passed to the decision-directed phase detector that derives a frequency control signal for a controlled oscillator, which in turn compensates the residual CFO [13].

\subsection{Receiver processing schedule}

At the start of a packet, the analogue front-end wakes up and performs automatic gain control (AGC). In the middle of the short preamble, the baseband receiver starts to perform coarse carrier synchronisation and symbol boundary detection. The coarsely estimated CFO is compensated before the DFT operation. The correlator bank then refines the symbol boundary selection. Two frequency-domain long preamble symbols generated by the DFT module are averaged, from which the frequency-domain equaliser coefficients are derived. Then the SIGNAL and data symbols can be demodulated accordingly. Meanwhile, the compensation for the offset between coarse and fine symbol boundaries is incorporated in the estimated channel gain. After that the receiver applies a QAM slicer to decide the output bits and also performs the non-data-aided decisiondirected phase detection to track the residual CFO in the frequency domain.

\section{Architecture design}

According to the algorithm and scheduling described in the previous section, we will now present an architecture for the baseband receiver. We carefully arrange the function of each block and optimise the power consumption and hardware utilisation and come up with the receiver block diagram illustrated in Fig. 2. Although the baseband receiver is made up of carrier synchronisation, symbol synchronisation, fast fourier transform (FFT) and equalisation, there exists some joint estimation and compensation mechanism in the first two blocks. Therefore, we will discuss them together.

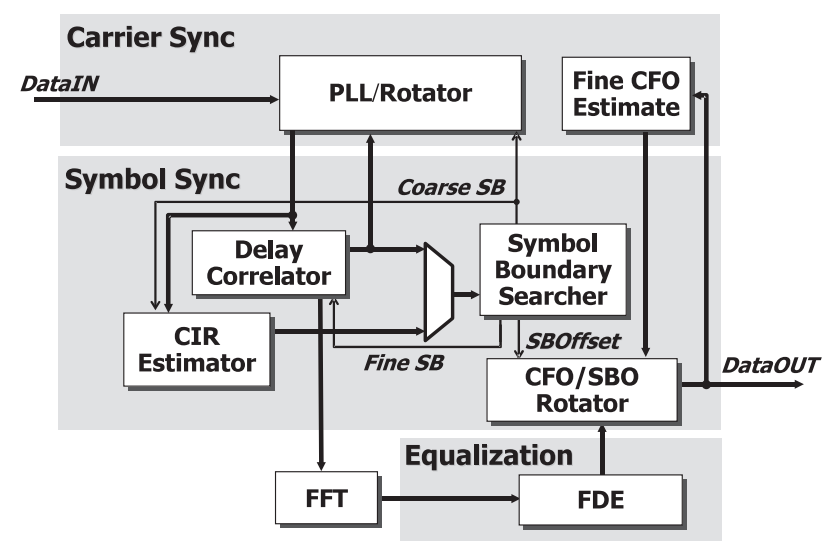

Fig. 2 Architecture of the proposed OFDM baseband receiver

\subsection{Carrier synchronisation and symbol synchronisation}

The carrier and symbol synchronisation blocks are shown in Fig. 3. The coarse carrier synchronisation block consists of the delay correlator and the PLL/rotator for coarse CFO estimation and time-domain compensation. The residual CFO is tracked by the CFO tracking block and compensated in the frequency domain. The symbol synchronisation part consists of the CIR estimator, the symbol boundary 


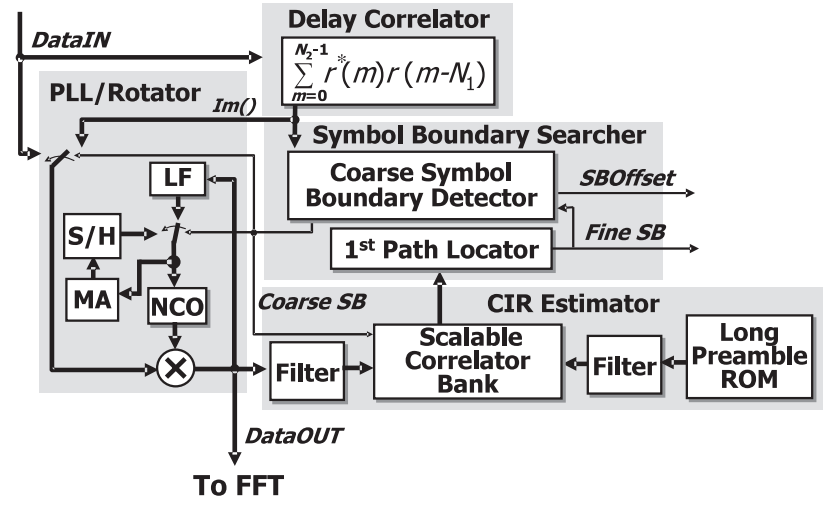

Fig. 3 Architecture of symbol boundary detection and coarse CFO synchronisation

searcher and also the delay correlator for coarse symbol boundary estimation.

3.1.1 Delay correlator: Since the period of short preamble is 16 samples, the delay can be any multiple of 16 . The delay correlator supports three different delay values; 16,32 and 64 . When receiving long preamble, the delay can be set to 64 for frequency-offset estimation. Moreover, a moving-average function is included with possible window lengths of 16,32 and 48 . The output of this block is used to detect the short preamble (start of the packet) and also to compute a coarse estimate of the $\mathrm{CFO}$ as explained in the following.

3.1.2 Phase locked loop/rotator: When the short preamble is being received, this block is configured as a second-order phase-locked loop (PLL) to track the phase of the delay-correlator output and the controlled oscillator control value can be used for estimating coarse CFO. In this way, no direct phase computation using the arc-tangent function is necessary. Another moving-average filter smooths the fluctuation in the estimated CFO. When the coarse symbol boundary is found, the output of the moving-average filter is sampled and held $(\mathrm{S} / \mathrm{H})$. This value is used by the numerically-controlled oscillator (NCO) to generate an oscillating sinusoidal waveform that compensates the estimated coarse CFO contained in the receiver input data.

\subsubsection{CIR estimator and symbol boundary} searcher: A bank of correlators, each made up of a multiplier, an accumulator and a squarer are used to estimate the channel impulse response when long preamble symbols start are being received (see Fig. 4). As the outputs of the correlators are the time-shifted version of the CIR, the more correlators, the wider the boundary search range.

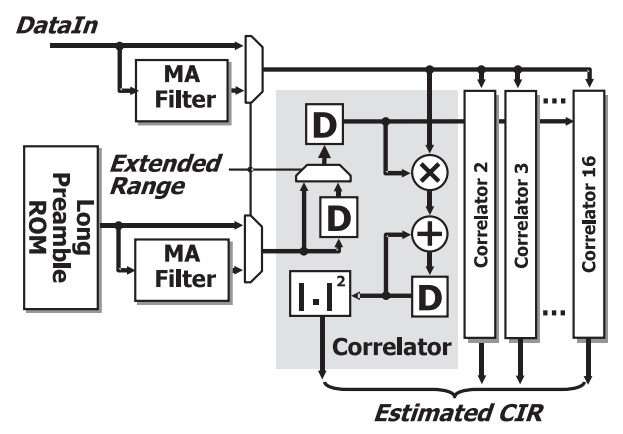

Fig. 4 Architecture of the time-domain channel impulse response estimator
To conserve hardware complexity and at the same time widen the search range, we design the correlator bank with an option of extended search range by spacing the timing of each correlator two samples apart rather than one sample. In this case, the search range will be doubled to 32 samples, while the symbol boundary can be accurate up to within two samples. Moreover, signals to be correlated are passed through two-point moving-average filters for smoothing. The criterion for detecting the fine symbol boundary is illustrated in Fig. 5. First a threshold proportional to the power of the estimated CIR is computed, then the first CIR sample that exceeds this threshold is deemed the fine symbol boundary. The difference between the coarse symbol boundary (detected by the delay correlator) and the fine symbol boundary (detected by the correlator bank) is the symbol boundary offset (SBOffset).

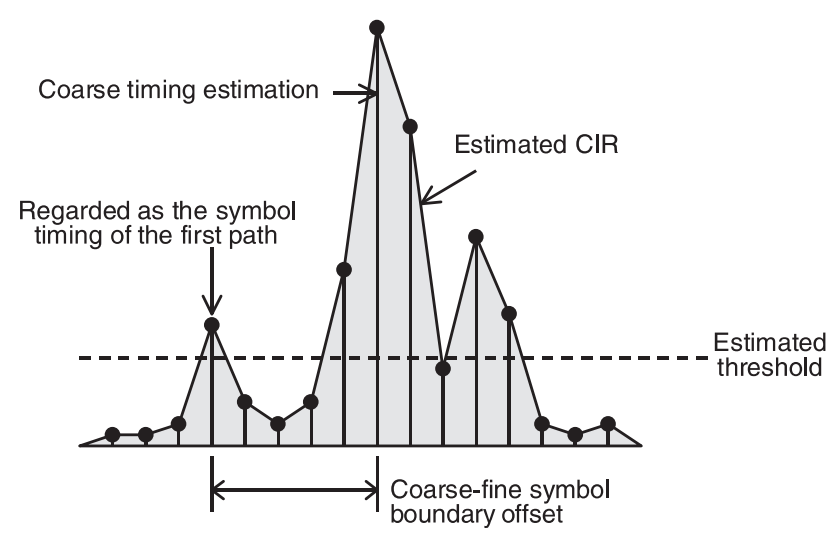

Fig. 5 Illustration of how the fine symbol boundary is determined

3.1.4 Fine CFO synchronisation: The coarse $\mathrm{CFO}$ estimated from short preamble can be imprecise and needs further fine $\mathrm{CFO}$ estimation and compensation. Figure 6 illustrates the block diagram of fine CFO synchronisation through a tracking loop. For CFO synchronisation, the decision-directed phase detector is used to both demodulate the QAM symbols and derive phase errors. The signals on deeply-faded subcarriers are too noisy and are excluded in the phase error computation.

In order to reduce latency, the receiver starts the FFT operation as soon as the coarse symbol boundary is found. Consequently, a symbol boundary offset exists and it induces constant phase shift in the frequency-domain signals. To compensate symbol boundary offset, a phase accumulator computes the corresponding phase shift compensation according to SBOffset. A phase rotator then

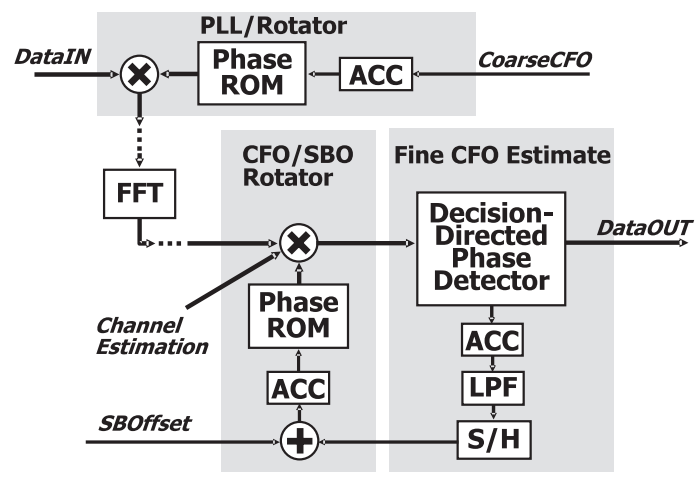

Fig. 6 Architecture of CFO tracking 


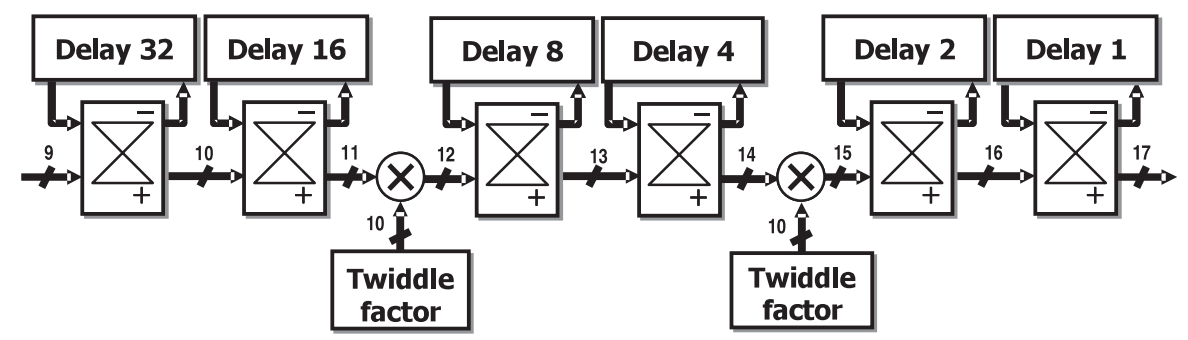

Fig. 7 Radix-2 $2^{2}$ FFT architecture

compensates the FFT outputs with phases computed from both the CFO loop and SBOffset. Note that this phase rotator is actually a complex-valued multiplier and in addition to phase rotation it also equalises the frequencydomain data by multiplying them with the equaliser coefficients obtained from channel estimation.

\subsection{FFT module}

FFT algorithms have been extensively studied and they can be categorised according to the radix used. Radix-2 FFT algorithm is by far the simplest to understand and to implement. An approach called radix $-2^{2}$ FFT algorithm retains the regular structure of the radix-2 FFT algorithm and cuts the number of complex multiplications in half [14]. Therefore, the proposed receiver adopts the radix $-2^{2}$ FFT algorithm. Two classes of FFT architectures are popular in the literature; single-path delay feedback (SPDF) [15] and multi-path delay commutator (MPDC) [16]. Selection of the FFT architecture is a tradeoff between cost, complexity and throughput. In this design, we choose the SPDF architecture and the radix $-2^{2}$ 64-point SPDF FFT architecture is shown in Fig. 7.

\subsection{Frequency-domain channel estimation and equalisation}

During the reception of long preamble, FFT outputs are divided by the long preamble subcarrier signals to estimate the channel gains of all subcarriers. In the case of a noisy and low delay-spread channel, we can compute a moving average of neighbouring subcarrier channel gains in order to get a more accurate channel gain estimation (see Fig. 8). After channel estimation, the subcarrier signals in data symbols are equalised in the frequency domain by multiplication of the reciprocal of their respective estimated channel gains.

The reciprocal operation is accomplished by the complex reciprocal module shown in Fig. 9. The reciprocal of a complex number $I_{i n}+j Q_{i n}$ takes the form of

$$
I_{\text {out }}+j Q_{\text {out }}=\frac{1}{I_{\text {in }}+j Q_{\text {in }}}=\frac{I_{\text {in }}-j Q_{\text {in }}}{I_{\text {in }}^{2}+Q_{\text {in }}^{2}}
$$

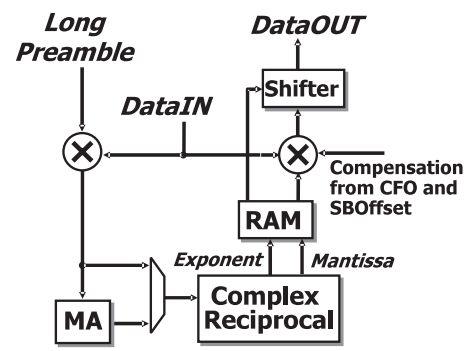

Fig. 8 Architecture of channel estimation and frequency domain equalisation

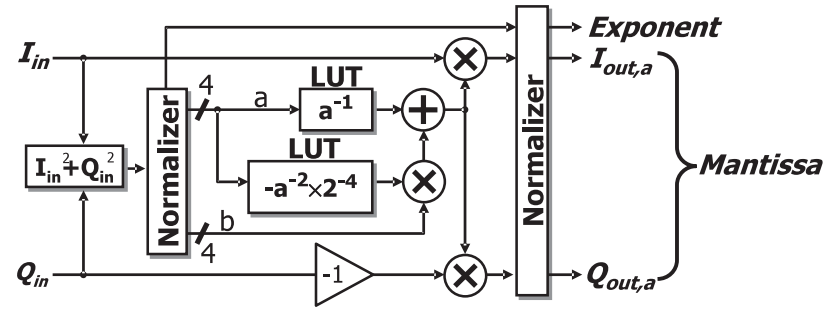

Fig. 9 Architecture of the complex reciprocal module

Note that an accurate implementation requires a division circuit, which is very costly especially when the dynamic range of the signal magnitude is large.

To this end, we propose an alternative approach to balance the hardware complexity and the system performance. At first we represent the power of the input in a floating-point number format, i.e. mantissa and exponent. The mantissa is further partitioned into groups of four-bit numbers

$$
I_{i n}^{2}+Q_{i n}^{2}=\left(a+b x+c x^{2}+d x^{3}+\cdots\right) \cdot 2^{E}
$$

where $8 \leq a \leq 15,0 \leq b, c, d \leq 15$ and $x=2^{-4}$ and $E$ is the exponent. The reciprocal of the mantissa can be expanded as a polynomial

$$
\frac{1}{a+b x+c x^{2}+d x^{3}+\cdots}=\frac{1}{a}+\frac{-b}{a^{2}} x+\cdots
$$

An approximation can be made by ignoring the higherorder terms. Considering the SNR requirement of the 64QAM constellation, we found that keeping the first two terms is good enough and the output of the complex reciprocal module is approximated by

$$
I_{\text {out }, a}+j Q_{\text {out }, a}=\left(I_{\text {in }}-j Q_{\text {in }}\right) \cdot\left(\frac{a-b x}{a^{2}}\right) \cdot 2^{-E}
$$

\subsection{Delay buffers}

In baseband signal processing systems many tasks have a need for delay buffers. Conceptually a $W$-bit delay buffer is made up by $W$ parallel flip-flop strings that propagate the incoming bits throughout all flip-flops. Since the flip-flopbased design is extremely power hungry, we use another delay buffer design that consumes much less power. Figure 10 shows a latch-based delay buffer design [17]. It is very similar to an SRAM design. An $N$-tap delay buffer consists of an ( $\mathrm{N}$-2)-column latch array, an address decoder and two extra pipeline registers. The address decoder is a flip-flop ring that controls the read-write address of the latch array. At any given time, it enables a column of latches for read and the next column for write. As most of the cells are not active, it consumes much lower power than the flip-flop-based design. The two pipeline registers are designed for input and output buffering to ensure that the module timing behaviour is the same as the flip-flop-based 


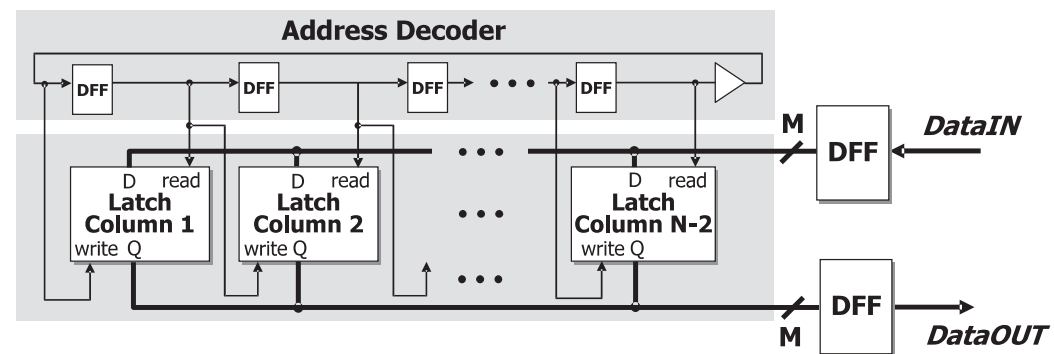

Fig. 10 Block diagram of the delay buffer

design. This architecture is highly scalable because all components of the module can be extended easily.

\section{Experimental results}

This chip is fabricated in a $0.35 \mu \mathrm{m}$ single-poly-quadruplemetal CMOS process and packaged in a 68-pin package. It is a cell-based design with many customised blocks (bit reversal module and delay buffers) to reduce both power consumption and active area. The transistor count is 429659 and the die measures $4102 \mu \mathrm{m} \times 4600 \mu \mathrm{m}$. Figure 11 shows the microphotograph of the receiver chip.

To simulate the channel effects, several exponential-decay channel profiles depicted in Fig. 12 are used to generate the simulated input signal for the receiver. The simulation results are averaged for performance evaluation. Moreover, channel profiles with stronger tails are selected to confirm the robustness of symbol boundary detection schemes. The functionality of the receiver chip is verified by a pattern generator and a logic analyser. Figure 13 plots the simulated and measured receiver performance under different delay spread with $100-\mathrm{kHz}$ carrier frequency offset. The signal-tonoise ratio (SNR) is defined as the ratio between OFDM data symbol energy and in-channel noise energy. The slight degradation in PER performance of the fabricated chip arises from the fact that a charge-sharing node in the chip sometimes makes the chip estimate the coarse CFO erroneously, and thus increases the measured PER.

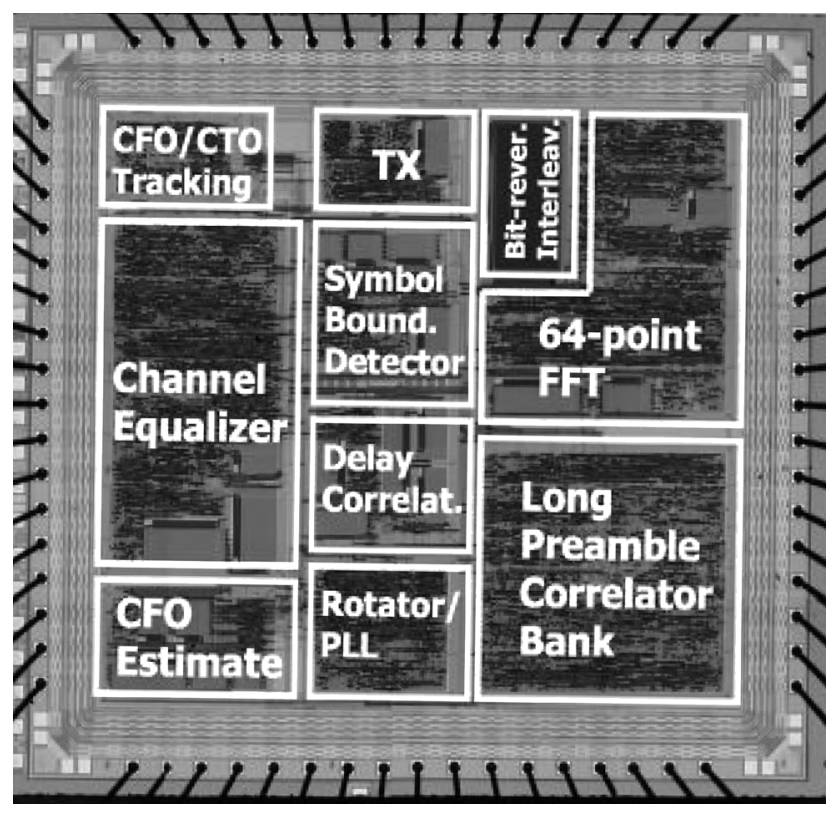

Fig. 11 Photograph of the receiver chip $\left(4.1 \times 4.6 \mathrm{~mm}^{2}\right)$

The power consumption of the chip is measured under different operation voltage when it is running at $20 \mathrm{MHz}$. The results are shown in Fig. 14. The chip consumes $168 \mathrm{~mW}$ at $3.3 \mathrm{~V}$ and $66 \mathrm{~mW}$ at $2.3 \mathrm{~V}$ supply voltage.
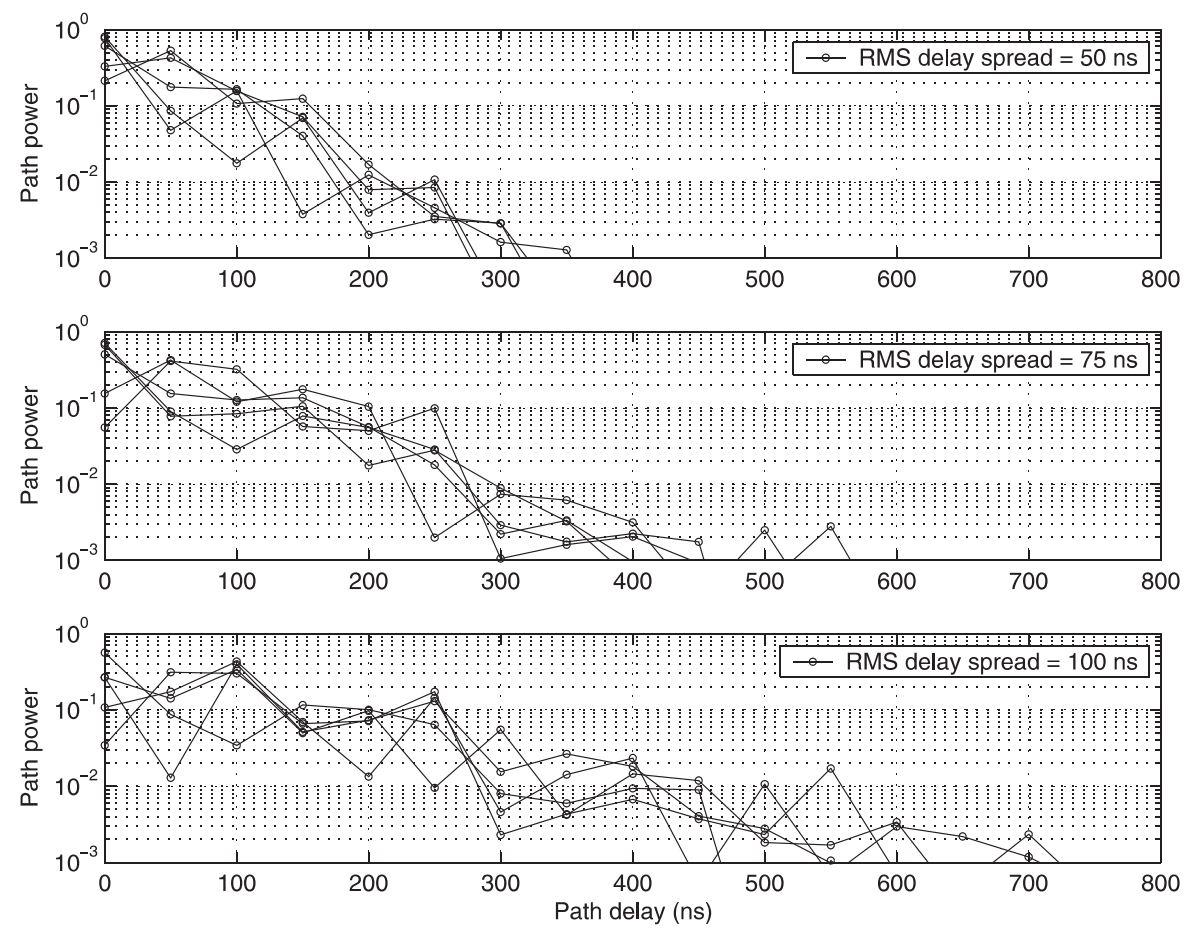

Fig. 12 Channel delay profiles used for receiver performance evaluation 


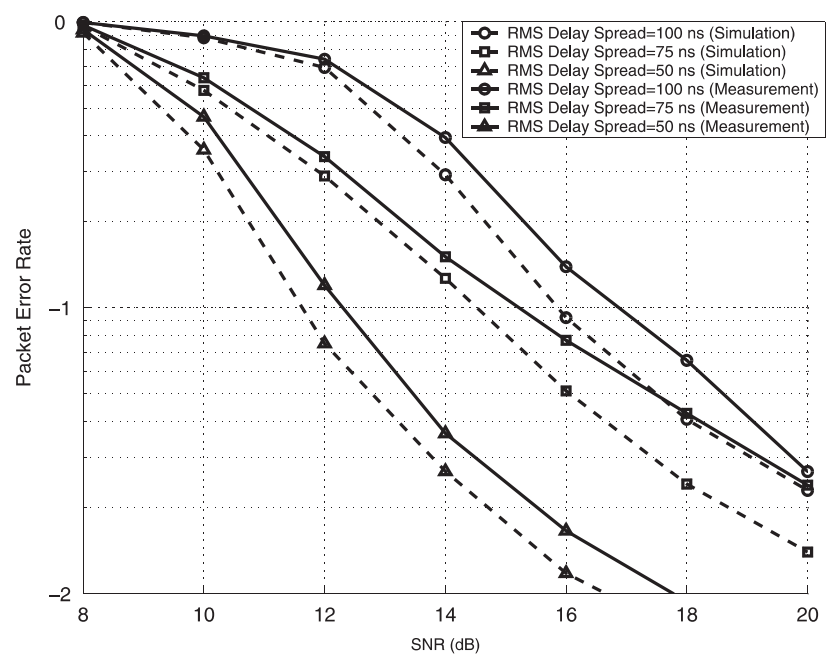

Fig. 13 Simulated and measured packet error rate (PER) performance of the OFDM receiver chip

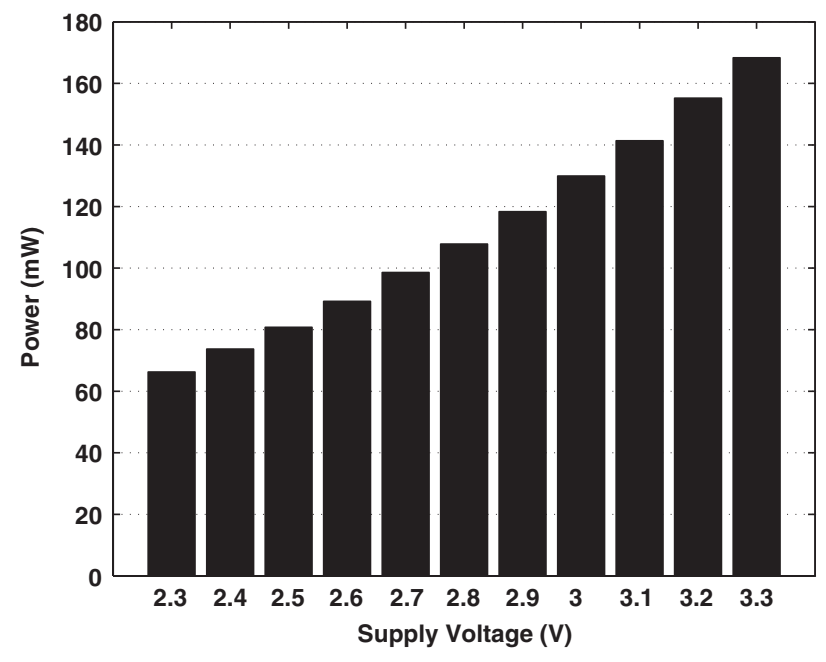

Fig. 14 Power consumption (including core and pad) of the proposed chip under different supply voltages

Table 1: Comparison with other OFDM WLAN baseband receiver chips

\begin{tabular}{llll}
\hline & Carnival [6] & Thomson [7] & Proposed IC \\
\hline Embedded A/D & not included & included & not included \\
Baseband processing & included & included & included \\
FEC and MAC modules & not included & included & not included \\
Clock rate $(\mathrm{MHz})$ & 20 & 80 & 20 \\
Technology $(\mu \mathrm{m})$ & 0.18 OLM & $0.251 \mathrm{P} 5 \mathrm{M}$ & 0.35 1P4M \\
Core voltage $(\mathrm{V})$ & 1.8 & 2.5 & 2.3 \\
$\begin{array}{l}\text { Die size }\left(\mathrm{mm}^{2}\right) \\
\text { Transistor count }\end{array}$ & 20.8 & 46.24 & 18.80 \\
(million) & 1.72 & 4.0 & 0.43 \\
RX Power $(\mathrm{mW})$ & 146 & 203 & 66 \\
\hline
\end{tabular}

A summary of the chip and its comparison with two OFDM-based WLAN receiver chips are listed in Table 1. The chip consumes less power than the other two, though using the least advanced technology. The chip has far fewer transistors, yet one other chip incorporates a channel decoder and medium access control (MAC) processor, making it more powerful. All in all, the proposed chip still provides a low-power solution to the baseband receiver implementation of the IEEE 802.11a/g OFDM-based WLAN systems.

\section{$5 \quad$ Receiver with space diversity}

Several receiving diversity schemes can be implemented based on the receiver chip that we have designed. In the following we compare the performance of several popular combining schemes such as selection combining (SC), equal gain combining (EGC) and maximum ratio combining (MRC) [11]. For simplicity, we only consider the case of a two-antenna diversity receiver. We use the same architecture of synchronisation blocks and the FFT block. QPSK modulation with $1 / 2$ rate convolutional code is used and the number of OFDM symbols is set to 50 per packet. We also take the average of the packet error rate over several channels with the same RMS delay spread. Channel profiles corresponding to different antennas are uncorrelated. Figure 15 shows the simulation results of packet error rates using different diversity techniques under multipath fading channel. The SNR in the figure denotes the signal-to-noise ratio per receiving antenna. It is clear that the MRC receiver with two-antenna diversity outperforms a one-antenna receiver by about $8-9 \mathrm{~dB}$. The performance of $\mathrm{SC}$ and EGC receivers is worse than for the MRC receiver. No matter which diversity technique is used, the curves with two antennas are steeper than the one with only one antenna. This is because the probability of signals at the same subcarrier from two antennas being both deeply faded is much less.

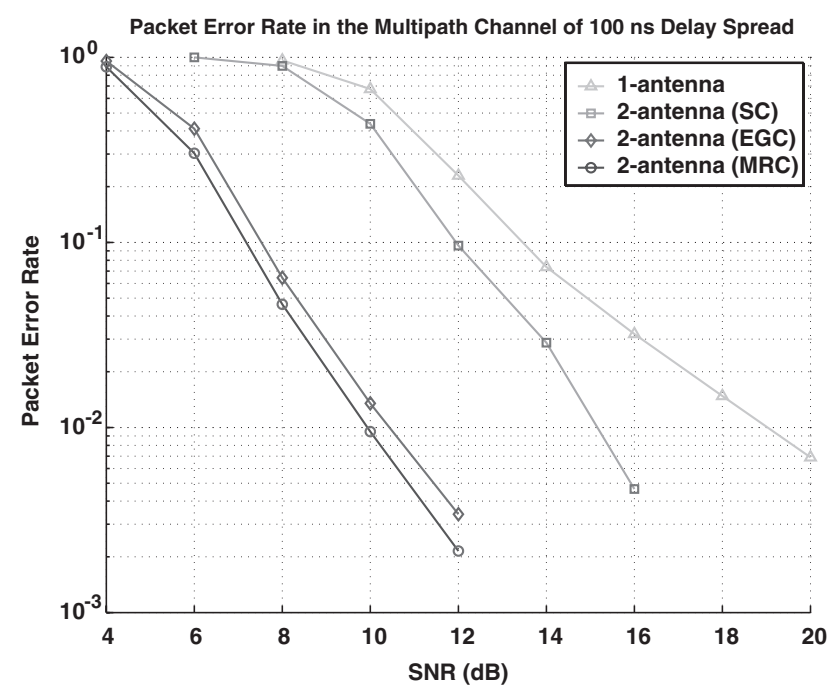

Fig. 15 Simulated packet error rate of receivers with two-antenna diversity using different space diversity schemes

Figure 16 illustrates the architecture of the MRC-based space diversity receiver. We use the symbol boundary detector, the CFO estimation and compensation block, and the FFT block in the receiver ASIC, and implement the other circuits, including post-FFT algorithms such as CFO tracking loops, frequency domain equalisation, diversity combining and the slicing circuit, using an FPGA. Figure 17 shows the measured and simulation results of packet error rates of the diversity receiver using the MRC technique under three multipath fading channels. The SNR in the figure denotes the signal-to-noise ratio per receiving antenna. There is about $1-\mathrm{dB}$ implementation loss between the simulated and the measured results due to the 


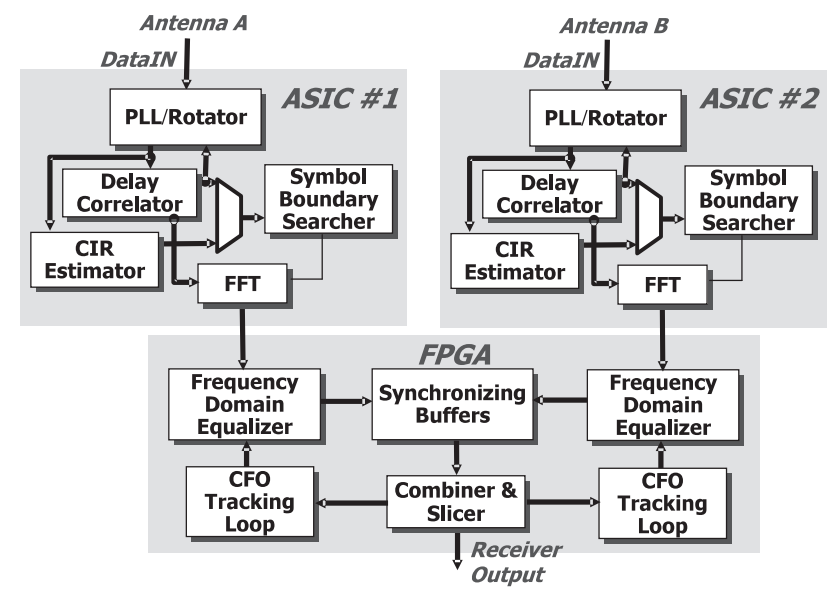

Fig. 16 Block diagram of the OFDM baseband receiver with space diversity

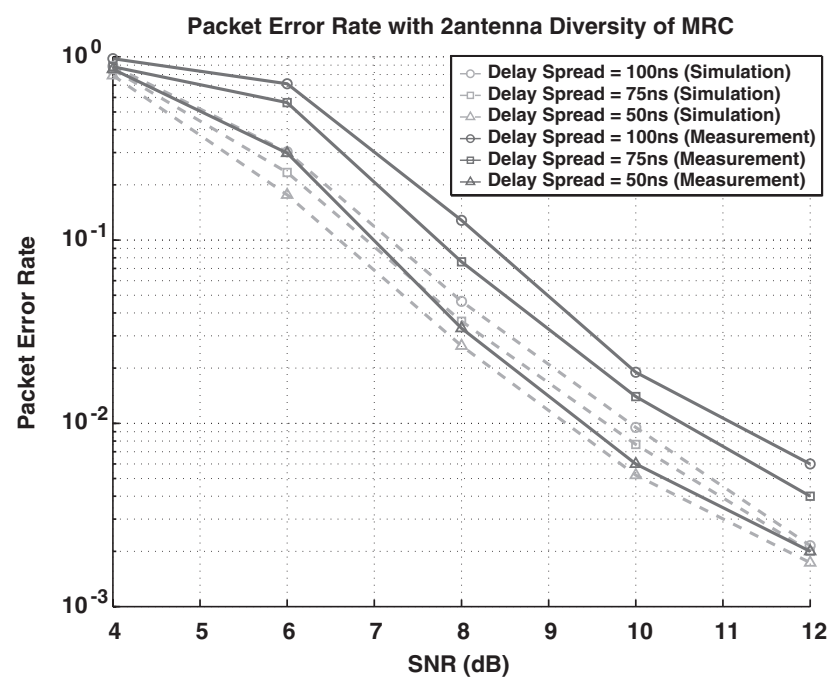

Fig. 17 Measured packet error rate of MRC-diversity receiver under channels with different delay spread

synchronisation issue between the baseband IC and the FPGA module. In summary, the space-diversity receiver outperforms the single-antenna receiver by about $5-8 \mathrm{~dB}$ in SNR and the effectiveness of the diversity receiver for OFDM-based wireless LAN systems in long delay-spread environments is confirmed.

\section{Conclusions}

In this paper, we have presented the design, implementation and measurement results of an OFDM space-diversity receiver for WLAN. We design a baseline OFDM baseband chip, and implement a space-diversity receiver based on the chip. The complexity and power consumption of the chip are minimised through innovative architecture, word-length optimisation and several circuit design techniques. The fabricated chip is then tested and shown to be functionally correct. Measurement results indicate that the power consumption of the proposed chip is as low as $66 \mathrm{~mW}$ running at $20 \mathrm{MHz}$ from a $2.3-\mathrm{V}$ supply voltage. Measured packet error rates show very good receiving performance under multipath fading channels. Moreover, the additional MRC scheme with two-antenna diversity outperforms a one-antenna receiver by about 5-8 dB, making it more suitable for situations with severe multipath fading.

\section{Acknowledgments}

The authors greatly appreciate the Chip Implementation Center (CIC), Taiwan for fabrication of the proposed chip. This work was supported in part by the National Science Council, Taiwan, ROC under grant no. 93-2220-E-002-013 and 93-2219-E-002-014.

\section{References}

1 Engels, M., Eberle, W., and Gyselinckx, B.: 'Implementation of a high performance wireless LAN'. Proc. IEEE Universal Personal Commun. Conf., 1994, pp. 253-256

2 Nee, R.V., and Prasad, R.: 'OFDM wireless multimedia communications' (Artech House, 2000)

3 Zou, W., and Wu, Y.: 'COFDM: an overview', IEEE Trans. Broadcast., 1995, 41, (1), pp. 1-8

4 'Wireless LAN medium access controol (MAC) and physical layer (PHY) specifications: High-speed physical layer in the $5 \mathrm{GHz}$ band'. IEEE Std 802.11a-1999, 1999

5 Rabaey, J.M., and Pedram, M.: 'Low power design methodologies' (Kluwer, Norwell, MA, 1995)

6 Eberle, W. et al: : $80-\mathrm{Mb} / \mathrm{s}$ QPSK and 72-Mb/s 64-QAM flexible and scalable digital OFDM transceiver ASICs for wireless local area networks in the 5-GHz band', IEEE J. Solid-State Circuits, 2001, 36, (11), pp. 1829-1838

7 Thomson, J. et al: 'An integrated 802.11a baseband and MAC processor'. Tech. Dig., IEEE Int. Solid State Circuits Conf. (ISSCC), 2002, Vol. 1, pp. 338-339

8 Liou, M.L., and Chiueh, T.D.: 'A parametric module design framework and its application to gate-level datapath/dsp mosule synthesis'. Proc. IEEE Int. Symp. on Circuits and Systems (ISCAS), May 2000, Vol. 2, pp. 41-44

9 Weinstein, S.B., and Ebert, P.M.: 'Data transmission by frequencydivision multiplexing using the discrete Fourier transform', IEEE Trans. Commun., 1971, 19, (5), pp. 628-634

10 Pollet, T., Bladel, M.V., and Moeneclaey, M.: 'BER sensitivity of OFDM systems to carrier frequency offset and Wiener phase noise', IEEE Trans. Commun., 1995, 43, (2), pp. 191-193

11 Rappaport, T.S.: 'Wireless communications, principles and practice' (Prentice Hall, Inc., NJ, 2002, 2nd edn.)

12 Yang, B., Letaief, K.B., Cheng, R.S., and Cao, Z.: 'Burst frame synchronization for OFDM transmission in multipath fading link' Proc. IEEE Veh. Tech. Conf., 1999, pp. 300-304

13 Nogami, H., and Nagashima, T.: 'A frequency and timing period acquisition technique for OFDM systems'. Proc. IEEE Personal, Indoor, Mobile Radio Commun., 1995, pp. 1010-1015

14 Shousheng, H., and Torkelson, M.: 'A new approach to pipeline FFT processor'. Parallel Processing Symp., Proc 10th Int. IPPS, Apr. 1996, pp. $766-770$

15 Wold, E.H., and Despain, A.M.: 'Pipeline and parallel-pipeline FFT processors for VLSI implementation', IEEE Trans. Comput., 1984, 33, (5), pp. 414426

16 Rabiner, L.R., and Gold, B.: 'Theory and application of digital signal processing' (Prentice Hall, Englewood Cliffs, NJ, 1975)

17 Ma, H.P., Liou, M.L., and Chiueh, T.D.: 'A 123-mW W-CDMA uplink basedband receiver IC with beamforming capability', IEEE $J$. Solid-State Circuits, 2004, 39, (5), pp. 785-794 